\title{
MULTIPLIERS BETWEEN SOME SPACES OF DISTRIBUTIONS
}

\author{
J. F. PRICE \\ (Received 28 August 1967; revised 17 June 1968). \\ Communicated by E. Strzelecki
}

By the systematic use of Fourier transforms and suitable weight functions L. R. Volevich and B. P. Paneyakh brought many classes of spaces of distributions (including the Sobolev spaces) and their topological duals under the one unifying definition. The main purpose here is to demonstrate that the representation of multipliers between pairs of these spaces (that is, continuous linear operators from one space into another which commute with translations) may be related to the representation of multipliers between $L^{p}$ and $L^{q}$. Also, complete representations of the multipliers from $\mathscr{D}$ and $\mathscr{S}$, the space of 'rapidly decreasing' functions, into $\mathscr{S}^{\prime}$, the space of temperate distributions, are given. The proofs of these results depend heavily upon the boundedness theorem 2.3 , which also provides a means of establishing new sufficient conditions for a distribution to be temperate.

\section{The spaces of distributions $H_{p}^{\mu}$}

Some familiarity with the elementary properties and topologies of the (distribution) spaces first described by Schwartz (see [9], I \& II) will be assumed. For example: $\mathscr{D}(\Omega)$, the inductive limit of Fréchet spaces $\mathscr{D}(K)$, $K$ ranging over compact subsets of $\Omega$, where $\Omega$ is an open subset of $R^{n}$, $\mathscr{D}^{\prime}(\Omega)$ and $\mathscr{D}^{m}(K)$ ([9], Chapitre I, $\S \S 2,3$; for convenience, we write $\mathscr{D}(K)$ in place of $\left(\mathscr{D}_{K}\right)$, etc.); $\mathscr{S}$ and $\mathscr{S}^{\prime}([9]$, Chapitre VII, $\S \S 3,4)$. As usual, $\mathscr{D}$ will denote $\mathscr{D}\left(R^{n}\right)$ and, unless stated otherwise, $\mathscr{D}^{\prime}(\Omega)$ and $\mathscr{S}^{\prime}$ will be considered to be equipped with their strong topologies.

If $1 \leqq p, q \leqq \infty$, denote by $L_{p}^{q}$ the set of $f \in \mathscr{S}^{\prime}$ satisfying

$$
\|f * \psi\|_{Q} \leqq \text { const. }\|\psi\|_{D} \text { for } \psi \in \mathscr{S},
$$

where $\|\cdot\|_{p}$ denotes the usual norm on the space $L^{p}=L^{p}\left(R^{n}\right)$. The space $L_{p}^{q}$, along with $M_{p}^{q}=\mathscr{F} L_{p}^{q}$, the space of Schwartz-Fourier transforms of elements in $L_{p}^{q}$, has been extensively studied with an aim to providing it with a more concrete description (see, for example, [3], [4] and [6]; the 
authors of [3] and [4] consider a locally compact Abelian Hausdorff topological group in place of $R^{n}$ ). The interest in $L_{p}^{q}$ will be made clear by [6], Theorem 1.2, which asserts that, if $T$ is a multiplier from $L^{p}$ into $L^{a}$, then there exists a unique $X \in L_{p}^{q}$ such that

$$
T \psi=X * \psi
$$

for $\psi \in \mathscr{S}$; and conversely, each $X \in L_{p}^{q}$ defines via (1.2) a multiplier from $\mathscr{S}$, equipped with the $L^{p}$-norm, into $L^{q}$.

A norm $\hat{f} \rightarrow M_{p}^{q}(\hat{f})$ is defined on $M_{p}^{q}$ as the norm of the map $\psi \rightarrow f * \psi$, where $f=\mathscr{F}^{-1} \hat{f}$, from $\mathscr{S}$, equipped with the $L^{p}$-norm, into $L^{q}$, This norm is precisely the smallest constant which may be used in (1.1).

In [6], Hörmander shows that $L_{p}^{q}=M_{p}^{q}=\{0\}$ for $p>q$ and that $M_{p}^{p} \subset M_{2}^{2}=L^{\infty}$.

Finally we come to the generalized function spaces $H_{p}^{\mu}$. These are essentially those defined and studied in [11], (Hörmander [7] also gives an independent discussion of the spaces $H_{2}^{\mu}$ ), although for a different class of weight functions since we will not require $H_{p}^{\mu}$ to be a module over $\mathscr{D}$, but will require that multiplication by the weight functions and their inverses define continuous isomorphisms from $\mathscr{S}$ onto itself. Denote by $\mathfrak{B}$ the class of $C^{\infty}\left(R^{n}\right)$-functions $\mu$ with the property that each of $\mu, \mu^{-1}$, $D^{r} \mu, D^{r} \mu^{-1}$ ( $r$ ranging over all indices) is of polynomial order at infinity.

1.1 Definition. $H_{p}^{\mu}=\left\{\psi \in \mathscr{S}^{\prime}: \varphi=\mathscr{F}^{-1}(\mu \psi) \in L^{p}\right\}$, where $\mu \in \mathfrak{B}$, and $\hat{\psi}=\mathscr{F} \psi$ denotes the Schwartz-Fourier transform of $\psi$.

1.2 Defining a norm on $H_{p}^{\mu}$ by $\|\psi\|_{p}^{\mu}=\|\varphi\|_{p}$ makes $H_{p}^{\mu}$ isometrically isomorphic to $L^{p}$. Also

$$
\mathscr{S} \subset H_{p}^{\mu} \subset \mathscr{S}^{\prime}
$$

where in both cases the embedded space is dense (provided $p \neq \infty$ in the first inclusion) in the enveloping space, and the inclusion maps are both continuous ([11], p. 67).

1.3 Example: The Sobolev space $W_{p}^{m}$. Consider the space $H_{p}^{\rho}$, where $1<p<\infty$ and $\rho(x)=\left(1+|x|^{2}\right)^{m / 2}, m$ being any non-negative integer. The Sobolev space $W_{p}^{m}$ is the space of temperate distributions which, along with their generalized derivatives of orders not exceeding $m$, belong to $L^{p}$. When $1 \leqq p \leqq \infty, W_{p}^{m}$ will be equipped with its usual norm, namely $f \rightarrow \sup \left\{\left\|D^{r} f\right\|_{p}:|r| \leqq m\right\}$. It follows easily from a sufficient condition of Mihlin, refined by Lizorkin (see [11], Lemma 13.1), that, for $|r| \leqq m$ and $1<p<\infty, x^{r} \rho^{-1} \in M_{p}^{p}$ where $r=\left(r_{1}, \cdots, r_{n}\right)$, an $n$-tuple of non-negative integers; $|r|=r_{1}+\cdots+r_{n}$; and $x^{r}=x_{1}^{r_{1}} \cdots x_{n}^{r_{n}}$. We will prove that, with $1<p<\infty$, the fact that $x^{r} \rho^{-1} \in M_{p}^{p}$ for $|r| \leqq m$ is equivalent to the statement that $H_{p}^{\rho}$ coincides, in the sense of topological vector spaces, with $W_{\rho}^{m}$. 
Proof. Calderón ([1], Theorem 7), for example, uses $x^{r} \rho^{-1} \in M_{p}^{p}$, $|r| \leqq m$, to prove that $H_{p}^{\rho}$ coincides with $W_{p}^{m}$. Alternatively, assume that $H_{p}^{\rho}$ and $W_{p}^{m}$ coincide. Let $\psi \in L^{p}$. Then $\mathscr{F}^{-1}\left(\rho^{-1} \hat{\psi}\right) \in H_{p}^{\rho}=W_{p}^{m}$ and so $D^{r}\left(\mathscr{F}^{-1}\left(\rho^{-1} \hat{\psi}\right)\right) \in L^{p}$ for $|r| \leqq m$, where $D^{r}=D_{1}^{r_{1}} \cdots D_{n}^{r_{n}}$ and $D_{i}$ denotes differentiation, in the sense of distributions, with respect to the $i$-th natural co-ordinate function on $R^{n}$. Now

$$
\mathscr{F}-1\left(x^{r} \rho^{-1}\right) * \psi=D^{r}\left((-i)^{|r|} \mathscr{F}-1 \rho^{-1}\right) * \psi=(-i)^{|r|} D^{r}\left(\mathscr{F}-1 \rho^{-1} * \psi\right)
$$

where $i^{2}=-1$. Applying the 'exchange formula' ([9], II. p. 124) to the last term shows that it equals $(-i)^{|r|} D^{r}\left(\mathscr{F}^{-1}\left(\rho^{-1} \hat{\psi}\right)\right)$ which, from the above discussion, belongs to $L^{p}$. To prove that $x^{r} \rho^{-1} \in M_{p}^{p}$, or equivalently, that $\mathscr{F}^{-1}\left(x^{r} \rho^{-1}\right) \in L_{p}^{p}$, it will suffice to show that the linear operator $w: \psi \rightarrow \mathscr{F}^{-1}\left(x^{r} \rho^{-1}\right) * \psi$ is continuous from $L^{p}$ into $L^{p}$ (see (1.1)). Since $w(\psi)=\mathscr{F}^{-1}\left(x^{r} \rho^{-1} \hat{\psi}\right)$ and $\mathscr{F}, \mathscr{F}^{-1}$ and multiplication by $C^{\infty}$-functions of polynomial order are continuous operators from $\mathscr{S}^{\prime}$ into $\mathscr{S}^{\prime}$ it follows that the graph of $w$ is closed. Banach's original version of the 'closed graph' theorem yields the required continuity of $w$.

As is commonly done, the normed dual of $W_{p}^{m}, 1 \leqq p<\infty$, will be denoted by $W_{p^{\prime}}^{-m}$, where $1 / p^{\prime}+1 / p=1$. When $1<p<\infty$, it follows readily that this dual is precisely $H_{p^{\prime}}^{\rho^{\prime}}$, where $\rho^{\prime}(x)=\rho^{-1}(x)=\left(1+|x|^{2}\right)^{-m / 2}$ (see [11], p. 66).

\section{Preliminary results on temperate distributions}

2.1 $\mathscr{F}$ (and $\mathscr{F}-1$ ) defines a continuous isomorphism from $\mathscr{S}$ and $\mathscr{S}^{\prime}$ onto $\mathscr{S}$ and $\mathscr{S}^{\prime}$ respectively ([9], Chapitre VII, $\S 6$ ). Multiplication by $\mu$ (and $\left.\mu^{-1}\right) \in \mathscr{B}$ is a continuous isomorphism from $\mathscr{S}$ onto itself. (This is evident from the definition of $\mathfrak{B}$.)

The topology of $\mathscr{S}$ may be defined by the sequence of seminorms

$$
S_{p}(\psi)=\sup \left\{(1+|x|)^{p}\left|D^{r} \psi(x)\right|:|r| \leqq p, x \in R^{n}\right\}
$$

for $p=0,1,2, \cdots$. Let $S_{p}^{\prime}$ denote the topological dual of $\mathscr{S}$ equipped with the norm $S_{p}(\cdot)$. The natural norm of $S_{p}^{\prime}$ will be denoted by $S_{p}^{\prime}(\cdot)$. It is not difficult to show that $\bigcup_{p=1}^{\infty} S_{p}^{\prime}=\mathscr{S}^{\prime}$. For completeness, we include the following known result.

2.2 Lemma. $A$ set $B$ in $\mathscr{S}^{\prime}$ is (weakly or strongly) bounded if and only if it is bounded in some $S_{m}^{\prime}$.

Proof. Since $\mathscr{S}$ is barrelled, every weakly bounded subset of $\mathscr{S}^{\prime}$ is equicontinuous ([2], 7.1.1), a fortiori strongly bounded. Let $B$ be a strongly bounded set in $\mathscr{S}^{\prime}$, that is

$$
\sup \{|\langle f, \psi\rangle|: f \in B, \psi \in U\}<\infty
$$


for any bounded set $U$ in $\mathscr{S}$. Then $B$ is bounded on some open non-void neighbourhood of 0 in $\mathscr{S}, V=\left\{\psi: S_{m}(\psi)<\delta\right\}$ say ([2], 0.3.18 and (2.1) above). The converse is evident.

2.3 TheOREM. Let $\mathscr{U}$ be a set of continuous linear operators from $\mathscr{D}(\Omega)$ into $\mathscr{S}^{\prime}$ which is (weakly or strongly) bounded at each point of $\mathscr{D}(\Omega)$, where $\Omega$ is an open subset of $R^{n}$. Then to each non-void compact subset $K$ of $\Omega$ there corresponds an integer $m$ such that $\mathscr{U}$ is equicontinuous from $\mathscr{D}(K)$, equipped with the $\mathscr{D}^{m}(K)$-norm, into $\mathscr{S}^{\prime}$.

Proof. Since $\mathscr{D}(\Omega)$ is the internal inductive limit of spaces $\mathscr{D}(\omega)$, $\omega$ a relatively compact subset of $\Omega$, the restrictions of each $u$ in $\mathscr{U}$ to $\mathscr{D}(K)$ are continuous. Now $\mathscr{D}(K)$ is a Fréchet space whose topology is defined by an increasing sequence of norms

$$
N_{p}(\psi)=\sup \left\{\left|D^{r} \psi(x)\right|:|\boldsymbol{\gamma}| \leqq m, x \in K\right\},
$$

$p=0,1,2, \cdots$. Since $\mathscr{S}^{\prime}=\bigcup_{p=1}^{\infty} S_{p}^{\prime}$, it follows that $\mathscr{S}^{\prime}=\bigcup_{p, q=1}^{\infty} q B_{p}$, where $B_{p}=\left\{f \in S_{p}^{\prime}: S_{p}^{\prime}(f) \leqq 1\right\}$. Now each $B_{p}$ is strongly (even weakly) closed in $\mathscr{S}^{\prime}$. (Let $\left\{f_{i}\right\}$ be a directed family in $B_{p}$ converging weakly in $\mathscr{S}^{\prime}$ to $f$; that is, $\langle f, \psi\rangle=\lim _{i}\left\langle f_{i}, \psi\right\rangle$ for each $\psi \in \mathscr{S}$. Then, for each index $i$, $\left|\left\langle f_{i}, \psi\right\rangle\right| \leqq S_{p}(\psi)$ for all $\psi \in \mathscr{S}$, and so $|\langle f, \psi\rangle| \leqq S_{p}(\psi)$ for all $\psi \in \mathscr{S}$; that is $f \in B_{p}$.) Thus $\left\{q B_{p}\right\}_{p, q=1}^{\infty}$ forms a countable, closed, convex, balanced covering of $\mathscr{S}^{\prime}$.

For each $q B_{p}$ we define

$$
V(q, p)=\bigcap\left\{u^{-1}\left(q B_{p}\right): u \in \mathscr{U}\right\} .
$$

Clearly the $V(q, p)$ are closed, convex and balanced. Moreover, since $\mathscr{U}$ is bounded at each point, they form a countable covering of $\mathscr{D}(K)$. (Let $\psi \in \mathscr{D}(K)$. Then $\mathscr{U}(\psi)=\{u \psi: u \in \mathscr{U}\}$ is bounded in $\mathscr{S}^{\prime}$ and so the previous lemma shows that this set must belong to some $q B_{p}$. Then $\psi$ belongs to the corresponding $V(q, p)$.)

Now $\mathscr{D}(K)$ is metrizable and complete so that, by the Baire category theorem, $\mathscr{D}(K)$ is non-meagre in itself. This assures the existence of positive integers $k, k^{\prime}$ such that $V\left(k^{\prime}, k\right)$ has non-void interior. So $\mathscr{U}$ maps a non-void open subset $V\left(\subset V\left(k^{\prime}, k\right)\right)$ of $\mathscr{D}(K)$ into a bounded subset of $\mathscr{S}^{\prime}$. $\left(\mathscr{U}(V)=\{u \psi: u \in \mathscr{U}, \psi \in V\}\right.$ is bounded since it is a subset of $k^{\prime} B_{k}$.) We may suppose $V$ is of the form $\psi_{0}+U$ where $U=\left\{\psi: N_{m}(\psi)<\lambda\right\}$. The set $\mathscr{U}\left(\psi_{\mathbf{0}}\right)$ is bounded in $\mathscr{S}^{\prime}$, by hypothesis, and therefore so is $\mathscr{U}(U)$. This implies that $\mathscr{U}$ is equicontinuous from $\mathscr{D}(K)$, equipped with the $\mathscr{D}^{m}(K)$ topology, into $\mathscr{S}^{\prime}$, since the fixed bounded set $\mathscr{U}(U)$ is, by definition, absorbed by any neighbourhood of 0 in $\mathscr{S}^{\prime}$. This completes the proof.

The following is an application of the above theorem which improves 
results of Schwartz (see [9]; VII, Théorème VI, $2^{\circ}$ et $3^{\circ}$ ) by giving weaker sufficient conditions for a distribution to be temperate.

2.4. Corollary. Let $X$ be a distribution and $\Omega$ some non-void open subset of $R^{n}$. Then for $X$ to be temperate it is necessary and sufficient that either

$$
\begin{aligned}
& X * \psi \in \mathscr{S}^{r} \quad \text { for each } \quad \psi \in \mathscr{D}(\Omega), \quad \text { or } \\
& X \hat{\psi} \in \mathscr{S}^{\prime} \quad \text { for each } \quad \psi \in \mathscr{D}(\Omega) \text {. }
\end{aligned}
$$

Proof. The necessity is obvious in both cases. Let $X * \psi \in \mathscr{S}^{\prime}$ $\left(X \hat{\psi} \in \mathscr{S}^{\prime}\right)$ for each $\psi \in \mathscr{D}(\Omega)$. Clearly this hypothesis will be satisfied for $\psi \in \mathscr{D}(h+\Omega)$, where $h \in R^{n}$, so that without loss of generality we may, and will, assume that $\Omega$ is a neighbourhood of zero. After introducing operators $u_{i}: \psi \rightarrow X_{i} * \psi \quad\left(v_{i}: \psi \rightarrow Y_{i} \hat{\psi}\right)$ where $X_{i}=X * \delta_{i} \quad\left(Y_{i}=X \hat{\delta}_{i}\right)$ belongs to $\mathscr{S}^{\prime}$ and $\left\{\delta_{i}\right\}_{i=1}^{\infty}$ is a regularizing sequence (see 3.1) in $\mathscr{D}(\Omega)$, the proof follows along exactly the same lines as that of Theorem 3.1, finally showing that $\left\{X_{i}\right\}\left(\left\{Y_{i}\right\}\right)$ is bounded in $\mathscr{S}^{\prime}$.

\section{Multipliers with range in $\mathscr{S}^{\prime}$}

3.1. Theorem. Let $T$ be a multiplier from $\mathscr{D}$ into $\mathscr{S}^{\prime}$. Then there exists a unique $X \in \mathscr{S}^{\prime}$ such that

$$
T \psi=X * \psi
$$

for $\psi \in \mathscr{D}$. Conversely, each $X \in \mathscr{S}^{\prime}$ defines a multiplier from $\mathscr{D}$ into $\mathscr{S}^{\prime}$ via (3.1).

Proof. The converse follows readily from well-known properties of the convolution operator, for example see Schwartz [9], Chapitre VII, Théorème $\mathrm{XI}$, and Chapitre VI, Theoreme IX.

Let $T$ be a multiplier from $\mathscr{D}$ into $\mathscr{S}^{\prime}$, and put $T \delta_{i}=X_{i} \in \mathscr{S}^{\prime}$, where $\left\{\delta_{i}\right\}$ is a regularizing sequence in $\mathscr{D}$; that is $\int_{R^{n}} \delta_{i}(x) d x=1$ and supp $\delta_{i} \rightarrow\{0\}$ as $i \rightarrow \infty$. Since $T$ is also a multiplier from $\mathscr{D}$ into $\mathscr{D}^{\prime}$ we learn from Edwards $[2], 5.11 .3$ that $T$ also commutes with convolutions. Thus

$$
T \psi=\lim _{i} T\left(\delta_{i} * \psi\right)=\lim _{i} X_{i} * \psi
$$

for each $\psi \in \mathscr{D}$. Let $T_{i} \psi=X_{i} * \psi$. Evidently the $T_{i}$ are continuous linear operators from $\mathscr{D}$ into $\mathscr{S}^{\prime}$, and satisfy the hypotheses of Theorem 2.3.

Let $K$ be any compact neighbourhood of 0 in $R^{n}$. Then there exists an integer $m$ such that $\left\{T_{i}\right\}$ is equicontinuous from $\mathscr{D}^{m}(K)$ into $\mathscr{S}^{\prime}$ (where $T_{i}$ also denotes the unique continuous extension of $T_{i}$ to all of $\left.\mathscr{D}^{m}(K)\right)$. By a suitable choice of a parametrix $\psi_{0} \in \mathscr{D}^{m}(K)$ (see $[9],(\mathrm{VI}, 6 ; 22$ ) or $[2]$, 5.11.2) we have 


$$
\Delta^{k} \psi_{0}=\delta-\varphi,
$$

where $\Delta^{k}$ is the iterated Laplacian, $\delta$ is the Dirac measure, and $\varphi \in \mathscr{D}(K)$. Thus

$$
X_{i}=X_{i} * \delta=X_{i} * \Delta^{k} \psi_{0}+X_{i} * \varphi=\Delta^{k} T_{i} \psi_{0}+T_{i} \varphi .
$$

Now $\left\{T_{i} \psi\right\}_{i=1}^{\infty}$ is bounded in $\mathscr{S}^{\prime}$ for each $\psi \in \mathscr{D}^{m}(K)$. (Let $U$ be a convex neighbourhood of 0 in $\mathscr{S}^{\prime}$ and let $\psi \in \mathscr{D}^{m}(K)$. Since $\mathscr{D}(K)$ is dense in $\mathscr{D}^{m}(K)$ and the $T_{i}$ are equicontinuous, then there exists $\psi_{1} \in \mathscr{D}(K)$ such that $T_{i} \psi-T_{i} \psi_{1} \in U$ for $i=1,2, \cdots$. But $\left\{T_{i} \psi_{1}\right\}_{i=1}^{\infty}$ is bounded in $\mathscr{S}^{\prime}$, whence there exists $\lambda>0$ such that $\left\{T_{i} \psi_{1}\right\} \subset \lambda U$, and so $T_{i} \psi \in U+\lambda U$ for $i=1,2, \cdots$. Since $U$ is convex, $U+\lambda U=(1+\lambda) U$, so that $\left\{T_{i} \psi\right\}$ is absorbed by $U$, and thus is bounded.) Thus (3.3) shows that the sequence $\left\{X_{i}\right\}$ is bounded in $\mathscr{S}^{\prime}$, is therefore strongly relatively compact $([2], 8.4 .7$ and 8.4.12), and so possesses a limit point, $X$ say. This completes the proof since the limit of $X_{i} * \psi$ in (3.2) can only be $X * \psi$.

3.2. Remarks. The multipliers $T$ from $\mathscr{S}$ into $\mathscr{S}^{\prime}$ are also represented exactly by (3.1) with $X \in \mathscr{S}^{\prime}$. This is evident from the initial references in the previous proof and the facts that $T \mid \mathscr{D}$ is a multiplier from $\mathscr{D}$ into $\mathscr{S}^{\prime}$, and $\mathscr{D}$ is dense in $\mathscr{S}$. This result will act as a sort of 'blanket' theorem for the following representations of multipliers between spaces of the form $H_{p}^{\mu}$.

\section{Representation of multipliers of $H_{p}^{\mu}$}

The proof of the theorem in this section uses the idea of $\left(\mathscr{S}^{\prime}\right)$-convolution introduced by Hirata and Ogata [5]; the $\left(\mathscr{S}^{\prime}\right)$-convolution of $f, g \in \mathscr{S}^{\prime}$ exists if and only if $\alpha, \beta \in \mathscr{S}$ implies $(f * \alpha) \cdot(\check{g} * \beta) \in L^{1}$. Of course the existence of the $\left(\mathscr{S}^{\prime}\right)$-convolution implies the existence of the Schwartz convolution. (See Shiraishi [10] for analogous definitions of the $\left(\mathscr{S}^{\prime}\right)$ convolution and Schwartz convolution.) The motivation behind the $\left(\mathscr{S}^{\prime}\right)$-convolution is that if the $\left(\mathscr{S}^{\prime}\right)$-convolution is defined between $f, g \in \mathscr{S}^{\prime}$, then the exchange formula is satisfied, that is

$$
(f * g)^{\wedge}=\hat{f} \cdot \hat{g} \text {. }
$$

4.1. The $\left(\mathscr{S}^{\prime}\right)$-convolution between $f \in L_{p}^{q}$ and $\phi \in L^{p}$ is defined, where $1 \leqq p, q \leqq \infty$, and belongs to $L^{q}$. If $p \neq \infty$, then $\phi \rightarrow f * \phi$ is continuous from $L^{p}$ into $L^{q}$.

Proof. Let $\alpha, \beta \in \mathscr{S}$. Then $f * \alpha \in L^{p} \cap L^{p^{\prime}}$, by (1.1), since $f \in L_{p}^{q}=L_{q^{\prime}}^{p^{\prime}}$ ([6], Theorem 1.3); and $\check{\phi} * \beta \in L^{p}$, by Young's inequality, so that $(f * \alpha) \cdot(\check{\phi} * \beta) \in L^{p^{\prime}} \cdot L^{p} \subset L^{1}$, as required. When $1 \leqq p<\infty$, the operator $\phi \rightarrow f * \phi$ is continuous from $L^{p}$ into $\mathscr{D}^{\prime}$ (Yoshinaga and Ogata [12], 
Theorem 3(2)). But from (1.1) we know that $\phi \rightarrow f * \phi$ for $\phi \in \mathscr{S}$ may be uniquely extended to a continuous operator from $L^{p}(1 \leqq p<\infty)$ into $L^{q}(1 \leqq q \leqq \infty)$. This extension must be $\phi \rightarrow f * \phi$ for $\phi \in L^{p}$, so that $f * \phi \in L^{q}$.

When $p=\infty$, we need only consider $q=\infty$, since $L_{p}^{q}=\{0\}$ when $p>q[10]$. First construct a sequence $\left\{\phi_{n}\right\} \subset \mathscr{D}$ which is bounded in $L^{\infty}$ and converges to $\phi$ weakly in $\mathscr{D}^{\prime}$. (Eg. $\varphi_{n}=f_{n} * \delta_{n}$, where $f_{n}=f$ for $|x| \leqq n$ and 0 otherwise.) Since $\left\{\phi_{n}\right\}$ is bounded in $L^{\infty},\left\{f * \phi_{n}\right\}$ is bounded in $L^{\infty}$, and thus is relatively weakly compact, so that it possesses a $\sigma\left(L^{\infty}, L^{1}\right)$-limit point in $L^{\infty}$. This limit point in $L^{\infty}$ can be none other than $f * \phi$.

4.2. THEOREM. Let $T$ be a multiplier from $H_{p}^{\mu}$ into $H_{a}^{\nu}$, where $\mu, \nu \in \mathfrak{B}$ and $1 \leqq p, q \leqq \infty$. Then there exists a unique $Y \in \mathscr{S}^{\prime}$ satisfying $\hat{Y} \in \mu / v M_{p}^{q}$ such that

$$
T \psi=Y * \psi
$$

for $\psi \in H_{p}^{\mu}$ when $p \neq \infty$, or $\psi \in \mathscr{S}$ when $p=\infty$. Conversely, $Y \in \mathscr{F}^{-1}\left(\mu / v M_{p}^{q}\right)$ defines a multiplier from $H_{p}^{\mu}$ when $p \neq \infty$, or $\mathscr{S}$ equipped with the $H_{p}^{\mu}$-norm when $p=\infty$, into $H_{a}^{\nu}$ via (4.2).

Proof. Throughout the proof we will assume $1 \leqq p<\infty$; the proof for $p=\infty$ follows with minor modification. We begin with the converse. Let $\psi \in H_{p}^{\mu}$, that is there exists $\phi \in L^{p}$ such that $\hat{\psi}=\mu^{-1} \hat{\phi}$; and let $Y \in \mathscr{F}^{-1}\left(\mu / \nu M_{p}^{q}\right)$, that is there exists $f \in L_{p}^{q}$ such that $\hat{Y}=\mu / v \hat{f}$. Let $\alpha, \beta \in \mathscr{S}$. Following the lines of the proof of 4.1 we have

$$
\psi * \alpha=\mathscr{F}^{-1}(\hat{\psi} \cdot \hat{\alpha})=\phi * \mathscr{F}^{-1}\left(\mu^{-1} \hat{\alpha}\right) \in L^{p},
$$

since $\mathscr{F}^{-1}\left(\mu^{-1} \hat{\alpha}\right) \in \mathscr{S}$ by 2.1 ; and $Y * \beta=f * \mathscr{F}-1(\mu / \nu \hat{\beta}) \in L^{p^{\prime}}$, since $f \in L_{p}^{q}=L_{q^{\prime}}^{p^{\prime}}$. Thus $(Y * \beta) \cdot(\psi * \alpha) \in L^{1}$ and the $\left(\mathscr{S}^{\prime}\right)$-convolution of $Y$ and $\psi$ is defined. Furthermore, application of (4.1) to $Y, \psi$, then to $f, \phi$, combined with 4.1 , yields

$$
\mathscr{F}^{-1}\left(\nu(Y * \psi)^{\wedge}\right)=\mathscr{F}^{-1}(\hat{f} \cdot \hat{\phi})=f * \phi \in L^{q},
$$

that is $Y * \psi \in H_{q}^{\nu}$. That the operator $\psi \rightarrow Y * \psi$ is continuous from $H_{p}^{\mu}$ into $H_{q}^{v}$ follows from (4.3) and the continuity of $\phi \rightarrow f * \phi$ from $L^{p}$ into $L^{q}$; while that it is linear and commutes with translations follows from the references in $\mathbf{3 . 1}$.

Let $T$ be a multiplier from $H_{p}^{\mu}$ into $H_{q}^{\nu}$. From 1.2, we know that $T \mid \mathscr{S}$ is a continuous linear operator from $\mathscr{S}$ into $\mathscr{S}^{\prime}$. Thus it satisfies the hypotheses of 3.2 and so there exists $Y \in \mathscr{S}^{\prime}$ such that (4.2) is valid for $\psi \in \mathscr{S}$. It remains to show that $Y \in \mu / \nu M_{p}^{q}$.

Now $T$ is continuous, and therefore bounded. We note from Remark 2 of Shiraishi [10] that the conditions of Hirata and Ogata [5] for the exchange 
formula to be valid are satisfied in the following manipulation, with the supremum taken over non-zero $\psi \in \mathscr{S}$;

$$
\begin{aligned}
& \|T\|=\sup \left\{\|Y * \psi\|_{q}^{\nu} /\|\psi\|_{p}^{\mu}\right\} \\
& =\sup \left\{\left\|\mathscr{F}^{-1}\left(\nu[Y * \psi]^{\wedge}\right)\right\|_{a} /\left\|\mathscr{F}^{-1}(\mu \hat{\psi})\right\|_{p}\right\} \quad \text { by definition, } \\
& =\sup \left\{\left\|\mathscr{F}^{-1}\left(\nu\left[Y * \mathscr{F}^{-1}\left(\mu^{-1} \hat{\psi}\right)\right]^{\wedge}\right)\right\|_{a}\|\| \psi \|_{p}\right\} \text { by 2.1, } \\
& =\sup \left\{\| \mathscr{F}-1\left(\mu / v Y \cdot \hat{\psi}\left\|_{q} /\right\| \psi \|_{p}\right\}\right. \\
& =\sup \left\{\left\|\mathscr{F}^{-1}(\nu / \mu \hat{Y}) * \psi\right\|_{q} /\|\psi\|_{p}\right\} .
\end{aligned}
$$

Since $v / \mu \hat{Y} \in \mathscr{S}^{\prime}$ by 2.1 , we have $M_{p}^{q}(\nu / \mu \hat{Y})=\|T\|$ by (4.4) and the definition of the norm of $M_{p}^{q}$. Thus $\nu / \mu \hat{Y} \in M_{p}^{q}$ and so we have the required $\hat{Y} \in \mu / v M_{p}^{q}$. If $p<\infty, \mathscr{S}$ is dense in $H_{p}^{\mu}$ (by (1.3)); and (4.2) for $\psi \in H_{p}^{\mu}$ is obtained as the continuous extension of $\psi \rightarrow Y * \psi$ for $\psi \in \mathscr{S}$.

REMARKs. (i) In particular cases it may be possible to obtain some information about $\mu / \nu$ from the following theorem, Theorem 13.1 of [11], 'For $H_{p}^{\mu}$ to be embedded in $H_{q}^{v}$ it is necessary and sufficient that $v / \mu \in M_{p}^{q}$ '.

(ii) In the previous proof we noted that $\alpha, \beta \in \mathscr{S}$ implied $\psi * \alpha \in L^{p}$ and $Y * \beta \in L^{p^{\prime}} \cap L^{p}$, where $\psi \in H_{p}^{\mu}$ and $Y \in \mathscr{F}^{-1}\left(\mu / \nu M_{p}^{q}\right)$. But this shows that $\psi \in \mathscr{D}_{L^{p}}^{\prime}$ and $Y \in \mathscr{D}_{L^{p}}^{\prime} \cap \mathscr{D}_{L^{p}}^{\prime}$ (see Schwartz [9], Chapitre VI, Théorème $\mathrm{XXV}, 2^{\circ}$ ) which provides an alternative verification that $Y * \psi$ exists ( $o p$. cit. Théorème XXVI, $2^{\circ}$ ).

The following specialization (ct. [6], Theorem 1.2 and [4], Theorem 2.1) of 4.1 to the Sobolev spaces mentioned in 1.3 is obvious since here $\mathscr{F}-1\left(\mu / v M_{p}^{q}\right)=L_{p}^{q}$. For $1<p \leqq 2,1 \leqq q \leqq 2$ and $m=0,1, \cdots$, this result was first proved by J. C. Merlo [8] using a completely different method which relied on a decomposition of elements in $L^{p}$.

4.3. Corollary. Let $T$ be a multiplier from $W_{p}^{m}$ into $W_{q}^{m}$, where $1<p, q<\infty$ and $m$ is any integer. Then there exists a unique $Y \in L_{p}^{q}$ such that (4.2) is valid for $\psi \in W_{p}^{m}$; and conversely.

REMARK. When $p=1$ or $\infty, 1 \leqq q \leqq \infty$, and $m$ is a positive integer, a sufficient condition for an operator from $\mathscr{S}$, equipped with the $W_{p}^{m}$-norm, into $W_{q}^{m}$ to be a multiplier is that it is of the form (4.2) with $Y \in L_{p}^{q}$. This follows readily by noting the continuity from $\mathscr{S}$ with the $L^{p}$-norm into $L^{q}$ of $D^{r} f \rightarrow Y * D^{r} f=D^{r}(Y * f)$ for each $r:|r| \leqq m$. An unpublished result of I. W. Wright shows that the condition is necessary when $p=1$, $1 \leqq q<\infty$ and $n=1$, but no necessary conditions of this nature are known for higher dimensions when $p$ is 1 or $\infty$.

The author wishes to thank his supervisor, Dr R. E. Edwards, for discussions and helpful comments during the preparation of this paper, and for suggesting the final version of Theorem 2.3. 


\section{References}

[1] A. P. Calderón, 'Lebesgue spaces of differentiable functions and distributions'. Proc. Sympos. Pure Math. IV. A.M.S. (1961), 33-49.

[2] R. E. Edwards, Functional Analysis: Theory and Applications. Holt, Rinehart and Winston, Inc. (1965).

[3] A. Figà-Talamanca and G. I. Gaudry, 'Density and representation theorems for multipliers of type $(p, q)^{\prime} . J$. Australian Math. Soc. 7 (1967), $1-6$.

[4] G. I. Gaudry, 'Multipliers of type $(p, q)$ '. Pacific J. Math. 18 (1966), 477-488.

[5] Y. Hirata and H. Ogata, 'On the exchange formula for distributions'. J. Sci. of Hiroshima Univ. Ser $A, 22$ (1959), 147-152.

[6] L. Hörmander, 'Estimates for translation invariant operators in $L^{p}$ spaces'. Acta Math. 104 (1960), 93-104.

[7] L. Hörmander, Linear Partial Differential Operators. Springer Verlag, Berlin (1963).

[8] J. C. Merlo, 'Some remarks on multipliers and their application to singular kernels'. (Spanish) Rev. Un. Mat. Argentina 20 (1962), 210-230.

[9] L. Schwartz, Théorie des Distributions, I et II. Hermann, Paris (1950-51).

[10] R. Shiraishi, 'On the definition of convolutions for distributions'. J. Sci of Hiroshima Uni. Ser $A, 23$ (1959), $19-32$.

[11] L. R. Volevich and B. P. Paneyakh, 'Certain spaces of generalized functions and embedding theorems'. Russian Math. Surveys, 20 (1965), 1-74.

[12] K. Yoshinaga and H. Ogata, 'On convolutions'. J. Sci. of Hiroshima Uni. Ser A, 22 (1958), $15-24$.

Institute of Advanced Studies

Australian National University 